\title{
TIME-SENSITIVE BEHAVIOR DYNAMICS IN MULTIMEDIA FINGERPRINTING SOCIAL NETWORKS
}

\author{
W. Sabrina Lin*, H. Vicky Zhao ${ }^{\dagger}$ and K. J. Ray Liu* \\ * ECE Dept., University of Maryland, College Park, MD 20742 USA \\ ${ }^{\dagger}$ ECE Dept., University of Alberta, Edmonton, AB T6G 2V4 Canada
}

\begin{abstract}
Multimedia social network is a network infrastructure in which the social network members share multimedia contents with all different purposes. Analyzing user behavior in multimedia social networks help design more secured and efficient multimedia and networking systems. In this paper, we focus on the colluder social network in multimedia fingerprinting systems in which colluders gain reward by redistributing the colluded multimedia signal. However, the market value of the redistributed multimedia content is time sensitive. The earlier the colluded copy being released, the more the people who are willing to pay for it. Thus the colluders have to reach agreement on how to distribute reward and the probability being detected among themselves as soon as possible. This paper incorporates the time-sensitiveness of the colluders' reward, and models the dynamics among colluders as a noncooperative game, and studies the time-restricted bargaining equilibrium. We provide the solution to the equilibrium that all the colluders have no inventive to disagree in order to maximize their own payoff.
\end{abstract}

Index Terms - Multimedia social network, game theory, behavior analysis

\section{INTRODUCTION}

A social network is a social structure made individuals or organizations that are tied by one or more specific types of interdependency. Users in a multimedia social network share and exchange multimedia contents, as well as other resources. Each member in a multimedia social network aims to maximize his/her own payoff, and different users have different objectives which are often conflicting with other users' objectives. In such scenario, Game theory $[1,2]$ provides a fundamental tool to study the behavior dynamics among multimedia social network members. By analyzing the human behavior in multimedia social networks, both the users and the system designer will have a clear picture of what is the reward every user can get in this multimedia social network, thus ultimately lead to systems with more secure, efficient and personalized services.

In this paper, without loss of generality, we use the multimedia fingerprinting system to illustrate the modelling and

The authors can be reached at wylin@eng.umd.edu, vzhao@ece.ualberta.ca, and kjrliu@eng.umd.edu. analysis of user behavior in multimedia social networks. Digital fingerprinting tracks the distribution of multimedia data to protect multimedia from illegal usage by embedding a unique label, known as fingerprint, into every distributed copy $[3,4]$. Multi-user collusion is a powerful attack against digital fingerprinting system, where a group of attackers collectively mount attack to effectively remove or attenuate the identifying information.

In the colluder social network, users collaborate with each other to reduce their chance of being caught by the digital right enforcer and share the reward of redistributing the colluded multimedia signal. One specific property of multimedia signals is that their market value is very time-sensitive. For instance, if the pirated version of a movie is released when the movie is in theater, it would have much higher market price than if it is released after the movie is available at DVD rental stores. Therefore, all the colluders have the incentive to mount collusion as soon as possible. Since the total reward of redistributing the multimedia signal is the sum of all colluders' reward, the users in the colluder social network have to agree on how to distribute the risk and reward and achieve agreement of the collusion attack. The prior work [5] considers the reward being a constant over time, thus the colluders can bargain for infinite stages to reach the equilibrium under different fairness constraints. However, since the reward is time-sensitive, the colluders must reach agreement within a few stages to release the colluded copy with high market value.

To address the time-sensitive property of multimedia signal and analyze the dynamics among the members in colluder social network, we model the user behavior as a noncooperative game where each colluder tries to maximize his/her individual payoff under the constraint that the agreement is made as soon as possible. We formulate the collusion as a time-sensitive bargaining process and find the equilibrium that maximize the colluders' payoff. The rest of the paper is as follows. Section 2 introduces the multimedia fingerprinting systems that we consider in this paper. We define the utility functions and the analyze time-restricted bargaining equilibrium in Section 3. We show simulation results in Section 4, and conclusions are drawn in Section 5. 


\section{SYSTEM MODEL}

\subsection{Scalable Video Coding Systems}

Scalable video coding is widely adopted nowadays to accommodate heterogenous networks and devices with different storage and computing capability. Scalable video coding decomposes the video sequence into different layers of different priority. The base layer contains the most important information of the video and is distributed to all users, and the enhancement layers gradually refine the reconstructed sequence at the decoder's side and are only distributed to the users with sufficient bandwidth. Without loss of generality, we consider a two-layer temporally scalable video coding system, where different frames are encoded at different layers [6]. For instance, in MPEG-2 video coding scheme, the base layer includes all the I frames, and the enhancement layer may contain all the $\mathrm{P}$ and $\mathrm{B}$ frames.

Define $F_{b}$ and $F_{e}$ as the sets containing the indices of the frames in base layer and enhancement layer, respectively; and let $F^{(i)}$ be the set of the indices of the frames that user $\mathbf{u}^{(i)}$ receives. $U^{b}$ is the subgroup of users who receive the base layer only, and $U^{b, e}$ is the set of all users who subscribe to the high quality version containing both layers.

\subsection{Scalable Multimedia Fingerprinting System}

Fingerprint Embedding We use the spread spectrum embedding [7] to embed fingerprints in the host signal. Let $\mathbf{S}_{j}$ be the $j$ th frame in the video, and for each user $\mathbf{u}^{(i)}$ who subscribes to frame $j$, the content owner generates a unique fingerprint $\mathbf{W}_{j}^{(i)}$, with the same length as $\mathbf{S}_{j}$. The fingerprinted frame is $\mathbf{X}_{j}^{(i)}=\mathbf{S}_{j}+J N D_{j} \mathbf{W}_{j}^{(i)}$, which is distributed to $\mathbf{u}^{(i)}$. $J N D$ [7] here is used to control the energy of the embedded fingerprints and make the fingerprinted copy be perceptually the same as the original one. In this paper, we first generate independent vector from Gaussian distribution $\mathcal{N}\left(0, \sigma_{w}^{2}\right)$, and then apply Gram-Schmidt orthogonalization to generate orthogonal fingerprints for different users.

Multi-user Collusion Since nonlinear collusion can be modelled as averaging collusion with additive noise and all collusion attacks have similar performance with colluded copies of the same quality [8], in this paper, we focus on averaging based collusion. Depending on the resolutions of the colluders' received copies, during collusion, the colluders are divided into two non-overlapping subgroups. $S C^{b}$ is the set including the indices of the colluders who receive the base layer only and $S C^{b, e}$ contains the indices of the colluders who subscribe to the high quality version which contains both base layer and enhancement layer. $K^{b}$ and $K^{b, e}$ are the number of colluders in $S C^{b}$ and $S C^{b, e}$, respectively, and $K=$ $K^{b}+K^{b, e}$ is the total number of colluders.

Without loss of generality, we consider the scenario where colluders who receive fingerprinted copies of the same resolution agree to share the same risk. Following the work in [6], colluders apply intra-group collusion first: for each frame $j \in F_{b}$ that they receive, colluders in $S C^{b}$ generate $\mathbf{Z}_{j}^{b}=$ $\sum_{k \in S C^{b}} \mathbf{X}_{j}^{(k)} / K^{b}$, and for each received frame $j \in F_{b} \cup F_{e}$, colluders in $S C^{b, e}$ calculate $\mathbf{Z}_{j}^{b, e}=\sum_{k \in S C^{b, e}} \mathbf{X}_{j}^{(k)} / K^{b, e}$. Then, the colluders apply inter-group collusion: for each frame $j \in F_{b}$ in the base layer, colluders generate $\mathbf{V}_{j}=\beta \mathbf{Z}_{j}^{b}+(1-$ $\beta) \mathbf{Z}_{j}^{b, e}+\mathbf{n}_{j}$ where $0 \leq \beta \leq 1$; and for each frame $j \in F_{e}$ in the enhancement layer, $\mathbf{V}_{j}=\mathbf{Z}_{j}^{b, e}+\mathbf{n}_{j} . \mathbf{n}_{j}$ is the additive noise to further deter the detection performance.

Fingerprint Detection: When identifying colluders, the fingerprint detector first extracts the fingerprint $\mathbf{Y}_{j}$ from frame $j$ for a the frames in the colluded copy. Then, for each user $\mathbf{u}^{(i)}$, the fingerprint detector calculates the detection statistics

$$
T N^{(i)}\left(\breve{F}^{(i)}\right)=\left(\sum_{j \in \breve{F}^{(i)}}\left\langle\mathbf{Y}_{j}, \mathbf{W}_{j}^{(i)}\right\rangle\right) / \sqrt{\sum_{j \in \breve{F}^{(i)}}\left\|\mathbf{W}_{j}^{(i)}\right\|^{2}},
$$

compares with a threshold $h$, and outputs the estimated colluder set $\widehat{S C}=\left\{i: T N^{(i)}>h\right\}$. When identifying colluders, the fingerprint detector can use fingerprints extracted from all layers collectively or examine each individual layer to determine whether a user is involved in collusion. For example, for user $i \in \mathbf{U}^{b, e}, \breve{F}^{(i)}$ has three choices, $F_{b} \cup F_{e}, F_{b}$ and $F_{e}$.

Here we adopt our prior work, the self-probing fingerprint in [9] as the fingerprint detector. The self-probing detector estimates the means of different detection statistics first, and then use the one with the largest estimated mean for identifying colluders. It was shown that the self-probing fingerprint detector has approximately the same performance as the optimum detector, which has perfect knowledge of the means and always select the detection statistics with the best performance.

\section{GAME MODEL OF HUMAN BEHAVIOR IN COLLUDERS' SOCIAL NETWORK}

In this section, we will first define the utility function of every member in the colluders' social network, find the feasible set of the game, and analyze possible bargaining solutions under different fairness criteria.

\subsection{Utility Function Definition}

During collusion, every member in the colluder social network wants to minimize his/her own risk and maximizes his/her own reward. For colluder $\mathbf{u}^{(i)}$, his/her payoff function $\pi^{(i)}$ should be composed of two terms: colluder $i$ 's loss if being detected plus his/her reward as follows:

$$
\pi^{(i)}=-P_{d}^{(i)} * L+R^{(i)}
$$

In (2), $P_{d}^{(i)}$ and $L$ stand for colluder $\mathbf{u}^{(i)}$ 's probability and loss of being detected, and $R^{(i)}$ is the profit that $\mathbf{u}^{(i)}$ gets by 
redistributing the colluded multimedia content.Since the total reward of redistributing the colluded copy is proportional to its quality: the better the quality is, the more total reward the colluders can get, and in temporal scalable video coding scenario, video quality is proportional to the number of frames, thus $R^{(i)}$ can be modelled as:

$$
R^{(i)}=\frac{F^{c} / F^{\max }}{\left[\sum_{j=1}^{K}\left(F^{(j)}\right)^{\gamma} D\left(P_{d}^{(j)}\right)\right] / M}\left(F^{(i)}\right)^{\gamma} D\left(P_{d}^{(i)}\right) .
$$

Where $F^{c}$ is the number of frames in the final colluded copy, and $F^{\max }$ is the largest number of frames among all the subscribers' copies, hence $F^{c} / F^{\max }$ illustrates higher quality of colluded copy gives higher reward to the colluders. $F^{(i)}$ is the number of frames in $\mathbf{u}^{(i)}$ 's copy; $\mathrm{K}$ is the total number of colluders, $\mathrm{M}$ is the total number of subscribers, and $D(\bullet)$ is a non-decreasing function. $\left(F^{(i)}\right)^{\gamma}$ illustrates colluders with higher-quality copies would share more reward, and $D\left(P_{d}^{(i)}\right)$ allows the colluders who take risk would have higher reward.

In the following sections, to simplify the analysis and without loss of generality, we assume the colluders who receive the same quality copies agree to share the same probability of being detected as in Section 2.2. Thus colluders who receive the low-resolution copies act as a single player in the game and they have the same utility $\pi^{b}$, while while colluders who have the high-resolution copies act as a single player during the bargaining process and they have the same utility $\pi^{b, e}$.

\subsection{Time-sensitive Bargaining Model}

The reward of redistributing the colluded multimedia signal depends on not only the colluded copy's quality, but also on the time that the copy being released. The market value of colluded copy with lower quality decreases faster than higherresolution copy. For instance, when the movie is still in theater, people might want to watch the low-resolution colluded copy to catch the trend. But if the movie is off theater and its DVD has been released, people might still want to purchase the high-resolution pirated copy since the cost would be lower than DVD, but the incentive of paying for low-resolution pirated version is very little, since the DVD is easily accessible and not very costy. Under this circumstance, both groups of colluders, $S C^{b}$ and $S C^{b, e}$ would want to reach agreement as soon as possible. We model the process of reaching agreement among colluders using a time-sensitive bargaining model

- In the first bargaining stage, $S C^{b, e}$ offers the collusion parameter $\beta_{1}$ that uniquely maps to the utility pair $\left(\pi_{1}^{b}, \pi_{1}^{b, e}\right)$ on the Pareto-optimal set, in which both $S C^{b}$ and $S C^{b, e}$ can not increase their payoff without decreasing the other's. The example of Pareto-optimal set is illustrated in Fig 1.

- Upon receiving the offer, $S C^{b}$ has the choice to accept

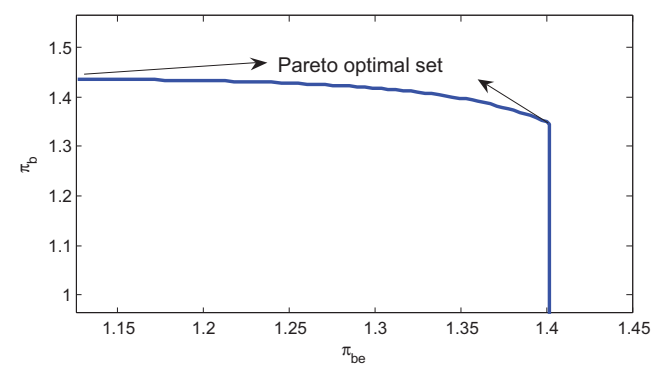

Fig. 1. An example of Pareto-optimal set for the bargaining problem

this offer and gets the payoff $\pi_{1}^{b}$, or reject and offer back $\beta_{2}$, which corresponds to payoff pair $\left(\pi_{2}^{b}, \pi_{2}^{b, e}\right)$ and continues to the second stage. The bargaining process would continue until both groups of colluders agree on one offer.

- Time-sensitive payoff function: If the colluders $S C^{b}$ and $S C^{b, e}$ reach agreement at the $k^{t h}$ bargaining stage, their reward would be decreased to $\delta_{b}^{k-1}$ and $\delta_{b, e}^{k-1}$ times, which means:

$$
\pi_{k}^{(i)}=-P_{d}^{(i)}\left(\beta_{k}\right) * L+\delta_{(i)}^{k-1} R^{(i)},
$$

where $(i) \in\{(b),(b, e)\}$ and $R^{(i)}$ as defined in (3).

In this model, $S C^{b, e}$ makes offer first and $\delta_{b, e} \geq \delta_{b}$ since colluders with higher resolution copies take advantage during bargaining. The equilibrium pair $\left(\left(\pi_{k}^{b}, \pi_{k}^{b, e}\right),\left(\pi_{k+}^{b} 1, \pi_{k+1}^{b, e}\right)\right.$ that the colluders reach agreement at the $k^{\text {th }}$ bargaining stage has the following property: suppose $S C^{b, e}$ makes an offer $\left(\pi_{k}^{b}, \pi_{k}^{b, e}\right)$ at the $k^{t h}$ stage, then $\pi_{k}^{b}$ should be large enough that $S C^{b}$ will accept it, and no larger. On the other hand, $S C^{b}$ should accept $\pi_{k}^{b}$ if it is not smaller than the discounted payoff $-P_{d}^{(i)}\left(\beta_{k+1}\right) * L+\delta_{(i)}^{k} R^{(i)}$ that $S C^{b}$ would receive if $S C^{b, e}$ accept their counter offer. Therefore,

$$
-P_{d}^{b}\left(\beta_{k}\right) * L+\delta_{b}^{k-1} R^{b}=-P_{d}^{b}\left(\beta_{k+1}\right) * L+\delta_{b}^{k} R^{b},
$$

and a similar consideration (for the dual game) for $S C^{b, e}$ gives

$-P_{d}^{b, e}\left(\beta_{k+1}\right) * L+\delta_{b, e}^{k} R^{b, e}=-P_{d}^{b, e}\left(\beta_{k}\right) * L+\delta_{b, e}^{k-1} R^{b, e}$.

We assume the worst-case scenario for the fingerprint detector that the colluders have perfect information about the detector's detection strategy. Thus $P_{d}^{b, e}(\beta)$ and $P_{d}^{b}(\beta)$ are known to the colluders. So we have two equations with two unknowns and the time-sensitive bargaining equilibrium can only be found numerically since $P_{d}^{b, e}(\beta)$ and $P_{d}^{b}(\beta)$ involve the Gaussian tail function. 


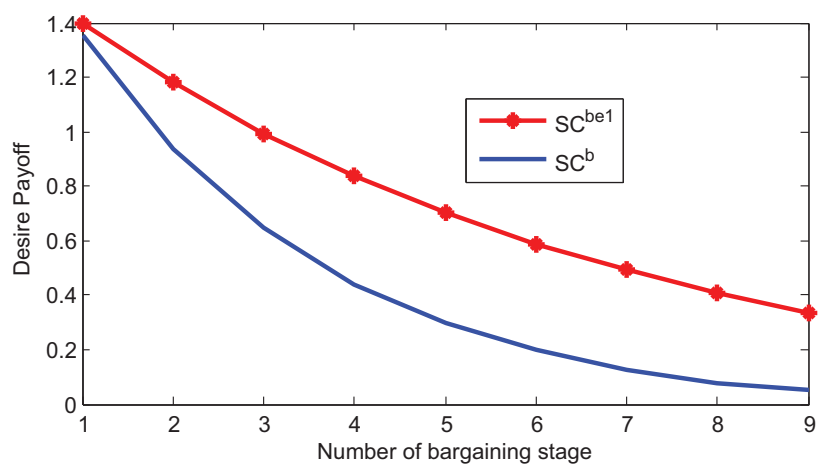

Fig. 2. Utilities of $S C_{b}$ and $S C^{b, e}$ versus number of bargaining rounds. $P_{f a}=10^{-3}, N_{b}=N_{e}=50000, K^{b}=100$, $K^{b, e}=150, \delta_{b}=0.7, \delta_{b, e}=0.85$, and $\left|U^{b}\right|=\left|U^{b, e}\right|=250$.

\section{CASE STUDY AND SIMULATION RESULTS}

In this section, we take a realistic utility functions as an example to illustrate the time-sensitive bargaining in the colluder social network. In real-world social networks, reward is usually distributed unequally because every member has different personal concern and position in the society, thus we consider the general utility function,

$$
\begin{aligned}
& \pi^{(i)}=-P_{d}^{(i)} * L+ \\
& \quad \frac{F^{c} / F^{\max }}{\left(K^{b}\left(F^{b}\right)^{0.1}+K^{b, e}\left(F^{b, e}\right)^{0.1}\right) / M}\left(F^{(i)}\right)^{0.1} P_{d}^{(i)},
\end{aligned}
$$

to illustrate the feasible region when the colluders distribute profit proportional to each copy's quality and the user's risk (probability of being detected).

\subsection{Simulation Setting and Results}

In our simulations, we first generate independent vectors following Gaussian distribution $\mathcal{N}(0,1)$, and then apply GramSchmidt orthogonalization to generate orthogonal fingerprints. The lengths of the fingerprints embedded in the base layer and the enhancement layer are $N_{b}=N_{e}=50000$, and both two layers contain 20 frames, respectively. The total number of users is 500, where $U^{b}=U^{b, e}$. The probability of accusing an innocent user, $P_{f a}$, is $10^{-3}$. Among the $K=250$ colluders, $K^{b}=100$ of them receive the fingerprinted base layer only, and the other $K^{b, e}=150$ of the colluders receive fingerprinted copies of high resolution.

Figure 2 shows the bargaining equilibrium versus the number of stages that the colluders need to make agreement Section 3.2 with utility function as in (7), and discount factor in (4) $\delta_{b}=0.7, \delta_{b, e}=0.85$. The feasible region and the Paretooptimal set with the same utility function for the first stage of the game is shown in Figure 1. It is clear from Figure 2 that both colluders have incentive to finish the bargaining process as soon as possible, especially for $S C^{b}$, who's utility decays much faster than $S C^{b, e}$. Therefore, at the very first bargaining stage, the first-mover will offer based on the equilibrium by solving (5) and (6). Thus $S C^{b}$ would let $S C^{b, e}$ to take the advantage of offering first. The discount factors $\delta_{b}, \delta_{b, e}$ can also be considered as the power of bargaining for $S C^{b}$ and $S C^{b, e}$. For instance, if the two groups of colluders cannot make agreement and they decide to collude within groups and generate 2 colluded copy with different qualities. Apparently $S C^{b}$ would get much less reward than $S C^{b, e}$ since their colluded copy has lower quality. Thus $S C^{b}$ has much more intention to cooperate with $S C^{b, e}$, and this intention leads to less bargaining power.

\section{CONCLUSIONS}

This paper studies the behavior modelling and analysis of the dynamics in colluder social network and address the special time-sensitive property of multimedia contents. We model the dynamics among colluders as a non-cooperative game, where each colluder aims to maximize his/her own utility through bargaining to reach agreement. Base on the general model of utility functions which allows unequal reward-distribution, we analyze the colluders' behavior by modelling collusion as a time-sensitive bargaining process and find the equilibrium of the bargaining game. Our analysis shows that in the colluder social network, the colluders will make agreement at the first bargaining stage and reach equilibrium. This paper provides a methodology that can fit human behavior analysis in different social networks.

\section{REFERENCES}

[1] G. Owen, Game Theory, Academic Press, 3rd edition, 1995.

[2] D. Fudenberg and J. Tirole, Game Theory, MIT Press, 1991.

[3] F. Hartung and B. Girod, "Watermarking of uncompressed and compressed video," Signal Processing, vol. 66, no. 3, pp. 283-301, 1998.

[4] K. J. R. Liu, W. Trappe, Z. J. Wang, M. Wu, and H. Zhao, Multimedia Fingerprinting Forensics for Traitor Tracing, EURASIP Book Series on Signal Processing and Communications, Hindawi Publishing Corporation, 2005.

[5] W. S. Lin, H. V. Zhao, and K. J. R. Liu, "Fairness dynamics in multimedia fingerprinting social networks," to appear, IEEE International conference on Image Processing, Oct 2008.

[6] H. Zhao and K. J. R. Liu, "Behavior forensics for scalable multiuser collusion: fairness versus effectiveness," IEEE Tran. on Information Forensics and Security, vol. 1, no. 3, pp. 311- 329, Sept. 2006.

[7] C. Podilchuk and W. Zeng, "Image adaptive watermarking using visual models," IEEE Journal on Sel. Area in Comm., vol. 16, no. 4, pp. 525540, May 1998.

[8] H. Zhao, M. Wu, Z. J. Wang, and K. J. R. Liu, "Forensic analysis of nonlinear collusion attacks for multimedia fingerprinting," IEEE Trans. on Image Processing, vol. 14, no. 5, pp. 646-661, May 2005.

[9] W. S. Lin, H. V. Zhao, and K. J. R. Liu, "Scalable multimedia fingerprinting forensics with side information," IEEE Int. Conf. on Image Processing, pp. 2293-2296, Oct. 2006. 